\title{
MULTICOMPONENT MASS TRANSFER DIFFUSION MODEL FOR THE ADSORPTION OF ACID DYES ON ACTIVATED CARBON
}

Keith K.H. Choy, John F. Porter and Gordon McKay

Department of Chemical Engineering, The Hong Kong University of Science and Technology, Clearwater Bay, Kowloon, Hong Kong SAR, China

The 3rd Pacific Basin Conference on Adsorption Science and Technology May 2003

\section{Abstract}

- The ability of activated carbon to adsorb two acid dyes, namely, Acid Blue 80 (AB80) and Acid Yellow (AY117) from wastewater has been studied in equilibrium and batch kinetic systems.

- A two resistance mass transport model based on film and surface diffusion control, Homogeneous Surface Diffusion Model (HSDM), has been applied to model the concentration decay curves in single component system.

- The effects of initial dye concentration and activated carbon mass on the rate of Acid Blue 80 and Acid Yellow 117 removal have been investigated.

- A multicomponent HSDM was developed to predict the binary component system decay curves from the single component HSDM and the IAST-RP model. 


\section{Introduction}

- Activated carbons are known to have extremely high surfaces areas (McKay, 1983).

- In addition, the adsorption capacity of acid dyes on activated carbon is relatively higher than that of several other adsorbents (Choy, 2001).

- Batch kinetic adsorption is an effective contacting operation which can provide a high degree of pollutant removal from effluents.

\section{Introduction}

Previous Work

- Mathews and Weber (1976) developed a solid-phase, homogeneous surface diffusion model (HSDM), using the finite difference solution method for any arbitrary isotherm in batch adsorption system.

- The HSDM has been successfully extended to batch kinetic adsorption systems on activated carbon in single component system. (Suidan et al., 1988; Miyahara and Okazaki, 1993; Traegner and Suidan, 1996; Axe et al., 1997; Trivedi et al., 2000 and McKay, 2001). 


\section{Introduction}

Present Work

- The semi-analytical solution method using the Redlich-Peterson (RP) equation has been applied to the acid dye batch kinetic adsorption system on activated carbon.

- A multicomponent HSDM for acid dye/carbon adsorption has been developed based on the ideal adsorbed solution theory (IAST) and the homogeneous surface diffusion model (HSDM) to predict the concentration versus time decay curves.

\section{Mathematical Model}

- The basic steps in this model include:

- the effect of external mass transfer

- unsteady-state surface diffusion in the particle

- a non-linear adsorption isotherm 


\section{Mathematical Model}

The assumptions of Multicomponent HSDM are as follows:

- The liquid film transport resistance occurs at the outer surface of the particle.

- The adsorbent particle is assumed to be a homogeneous solid in which the adsorbate is transported by surface diffusion;

- Pore diffusion makes a negligible contribution to mass transport.

\section{Model Equations}

The concentration of solute inside the typical particle at distance $r$ from the centre and at time $\mathrm{t}$ is defined by $q_{j}\left(r_{j}, t\right)$ and the variation of $q_{j}$ with distance and time is governed by the diffusion equation for each component $j$ :

A dimensionless variables are applied by defining $\varphi_{j} \equiv X_{j} q_{j}$, and in equation (1) to form equation (2) with the following boundary conditions (3) and (4).

(2)

(4) 


\section{Model Equations}

The material balance equation for the multicomponent system and the rate of change of solid phase concentration of each component $j$ are:

\section{(7)}

The mathematical problem presented by the model requires the simultaneous solution of equations (2) to (7). The starting point is a semi-analytical solution of equation (8), previously only applied to single component systems, that satisfies the boundary conditions in equations (3) and (4).

\section{Model Equations}

By considering a short time interval, $\tau_{0}, \tau_{1}, \tau_{2} \ldots, \tau_{n}$ in the integration of equation (8) for $j$ component and it is assumed that the time intervals are sufficiently short that is effectively constant in each interval to solve equation ( 8 ) by a numerical method. Also, equation (7) becomes:

The IAST with the Redlich-Peterson equation is used to determine the pair of liquid phase concentrations, $C_{s, 1}$ and $C_{s, 2}$ from the corresponding pair of solid phase concentrations, $q_{s, 1}$ and $q_{s, 2}$, at the surface of the carbon particle in the binary component. 


\section{Model Equations}

The IAS theory dictates that the spreading pressure should be constant for each component in a given system

For a given pair of $q_{e, 1}$ and $q_{e, 2}$ the values of $s_{1}, s_{2}$ amd $q_{T}$ can be calculated.

To calculate the spreading pressure, numerical computer integration is used to solve the equation (13).

\section{Model Equations}

For a given (guess values) pair of $q_{e, 1}$ and $q_{e, 2}$, the values of $S_{1}$, $S_{2}$ and $q_{T}$ can be calculated. Combining the Redlich-Peterson equation (14) and equation (12) gives equation (15).

A numerical computer program is used to optimize the value of until the spreading pressures of component 1 and 2 are equal. Hence, the value of $C_{e, 1}$ and $C_{e, 2}$ can be calculated from the and by equations (16) and (17) while and Consequently: 


\section{Materials}

Adsorbent

- The adsorbent used in this study is Filtrasorb 400 granular activated carbon (GAC) supplied by Chemviron Ltd.

- The GAC was washed in deionised water, dried and sieved to obtain certain size ranges: $355-500,500-710$ and 710-1000 microns.

Figure 1: Molecular Structure of Acid Blue 80

Adsorbate

The adsorbate used in the experiments is Acid Blue 80 (AB80) and Acid Yellow 117 (AY117) supplied by Ciba Specialty Chemicals.

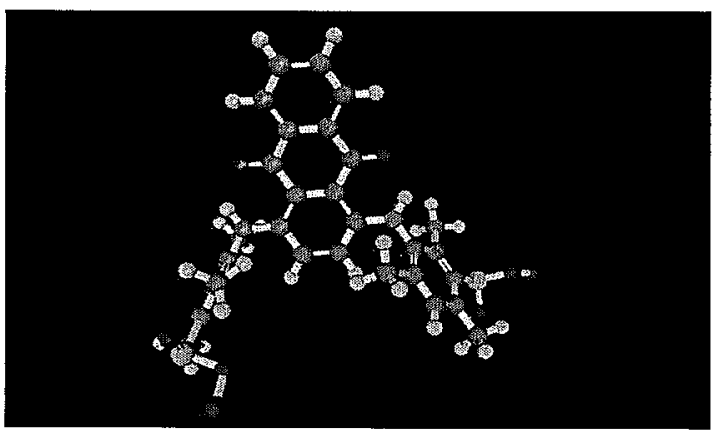

\section{Batch Kinetic Experimental System}

An adsorber vessel in a standard batch stirred tank configuration is used in the batch experiments.

- The impeller speed is $400 \mathrm{rpm}$.

- The temperature is kept at $20 \pm 2^{\circ} \mathrm{C}$.

- Volume of dye solution is $1.7 \mathrm{dm}^{3}$.

- Experimental contact time is $48 \mathrm{hrs}$.

The influence of initial dye concentration and carbon mass on ( $500-710 \mu \mathrm{m})$ the adsorption rate has been investigated.

- Different carbon masses: $0.4,0.8,1.2,1.7 \& 2.2 \mathrm{~g}$

- Different initial dye concentrations: $50,75,100,150 \&$ $200 \mathrm{ppm}$. 
Figure 2: Standard Tank Configuration for Batch Kinetic Studies

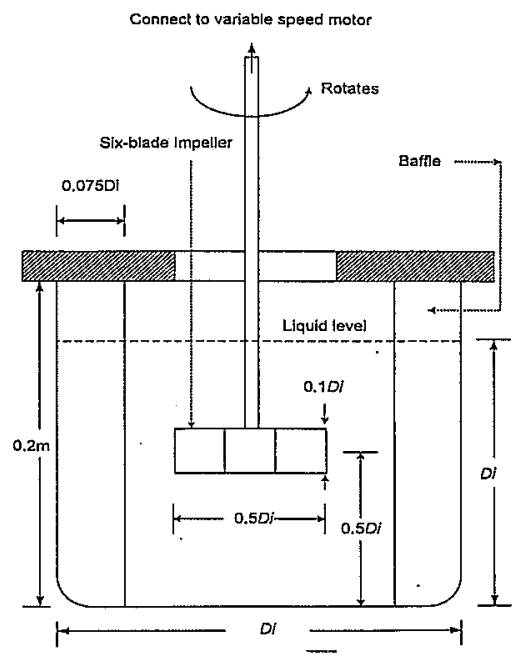

\section{Results and Discussion}

\section{Equilibrium Isotherm Studies}

The equilibrium isotherms were measured for Acid Blue 80 (AB80) and Acid Yellow 117 (AY117) on Activated Carbon F400. The Redlich-Peterson isotherm is used to relate equilibrium concentrations between liquid phase and solid phase loading. The isotherm parameters of $K_{R^{\prime}} a_{R}$ and $\beta$ for two acid dyes are shown in Table 1.

$$
\text { R-P equation : }
$$

Table 1: Redlich-Peterson Isotherm Constants for AB80 and AY117 in Single Component System

\begin{tabular}{|l|l|l|}
\hline & AB80 & AB80 \\
\hline $\boldsymbol{K}_{\boldsymbol{R}}\left(\mathbf{d m}^{\mathbf{3}} / \mathbf{g}\right)$ & 28.32 & 55.40 \\
\hline $\boldsymbol{a}_{\boldsymbol{R}}\left(\mathbf{d m}^{\mathbf{3}} / \mathbf{m m o l e}\right)^{\beta}$ & 103.6 & 200.2 \\
\hline $\boldsymbol{\beta}$ & 0.965 & 0.910 \\
\hline SSE Value $\left(\mathbf{1 0}^{-4}\right)$ & 1.088 & 1.191 \\
\hline
\end{tabular}


Figure 3: Redlich-Peterson Isotherm Plots for Two Acid Dyes in Single Component Systems

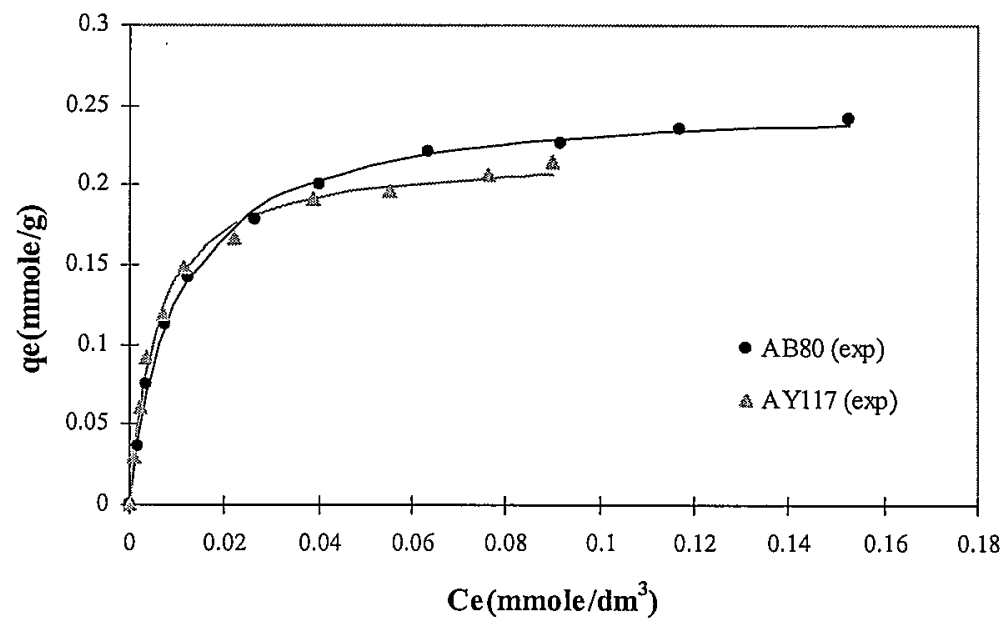

Figure 4: Redlich-Peterson Isotherm Plots for Two Acid Dyes in Binary Component Systems 


\section{Results and Discussion}

Batch Kinetic Studies

- The effects of initial solute concentration and adsorbent mass have been studied on the diffusion processes for the multicomponent HSDM.

- The HSDM was used to correlate two acid dyes (AB80 \& AY117) in the single component system in order to determine the relation between the single component and multicomponent systems.

- A comparison of the theoretical and experimental data for the AY117 in AB80+AY117 binary systems with different carbon masses is illustrated in Figure 5 and Figure 6.

Figure 5: Effect of Activated Carbon Mass on the Adsorption of $A B 80$ in $A B 80+A Y 117$ using Multicomponent HSDM (IAS-RP)

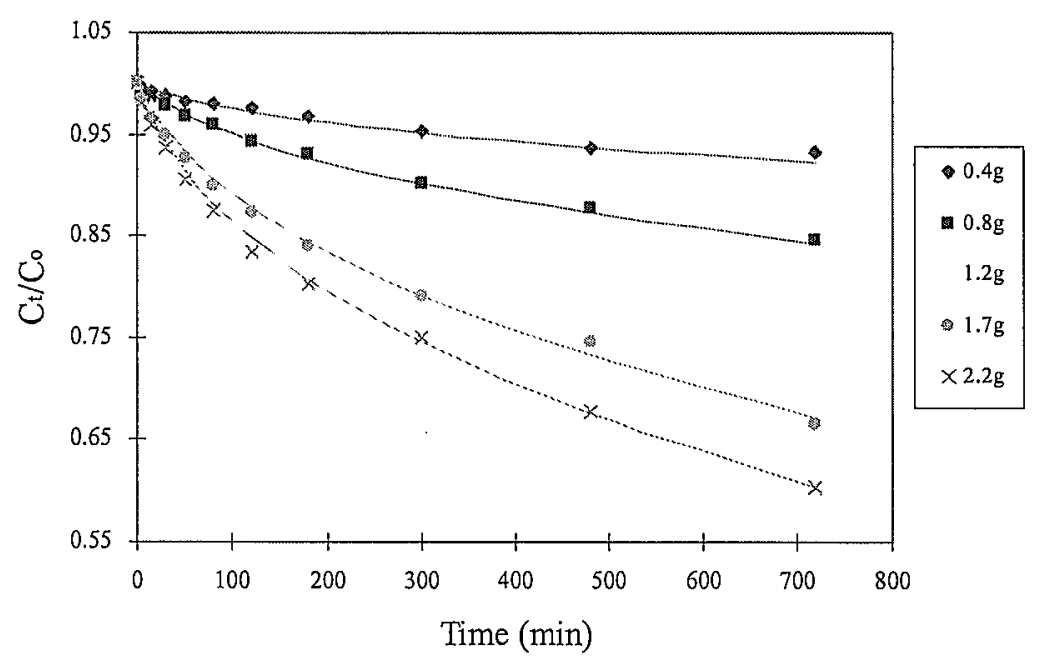


Figure 6: Effect of Initial Dye Concentrations on the Adsorption of AY117 in AB80+AY117 using Multicomponent HSDM (IAS-RP)

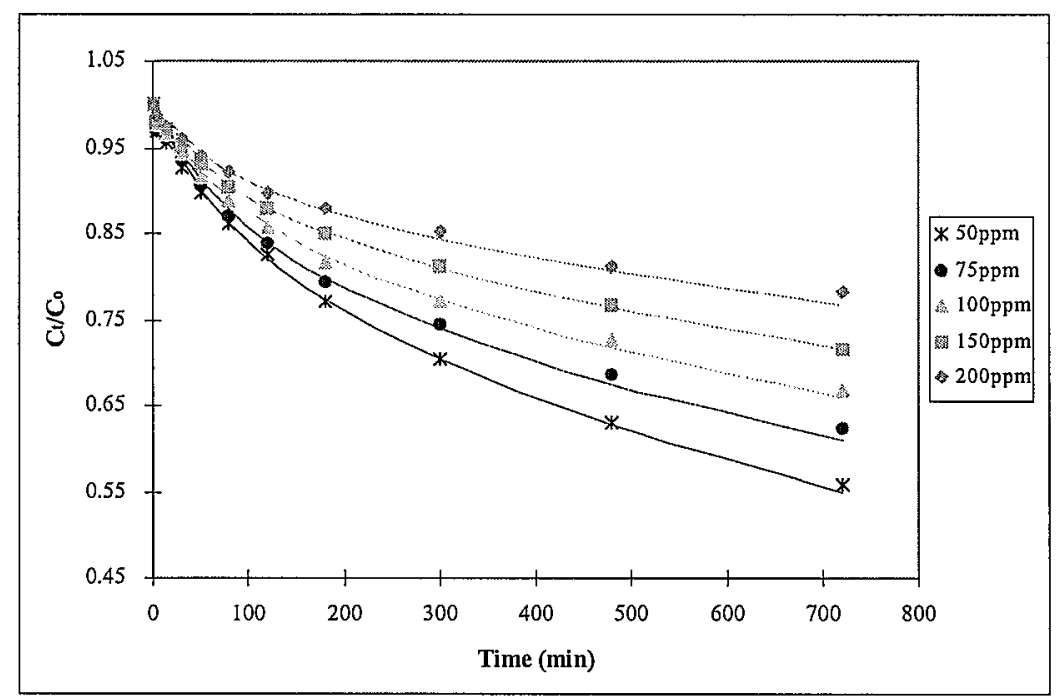

\section{Batch Kinetic Studies (Results and Discussion)}

- Single component external mass transfer coefficient, $k_{f 1}$ and $k_{f, 2}$, are used in the multicomponent HSDM model. $\mathrm{ft}$ was found that there is some deviation in the first 30 minutes for the two binary component systems.

- The results indicate that the values of the external mass transfer coefficient in the multicomponent system are slightly different to those of the single component system.

- The $D_{s, i}$ and SSE values for the AB80+AY117 binary system ${ }^{s, i}$ with different carbon masses and different initial concentrations are shown in Table 2.

- It was found that parameters $D_{s, 1}$ and $D_{s, 2}$ are almost constant for different carbon máss systems while the surface diffusion coefficients are strongly dependent on the initial dye concentration of each system. 
Table 2: External Mass Transfer and Solid-phase Diffusion Coefficients for the Adsorption of AB80+AY117 on Activated Carbon using the Multicomponent HSDM (IAS-RP).

\begin{tabular}{|c|c|c|c|c|c|c|}
\hline \multirow[t]{2}{*}{$\begin{array}{c}\text { Carbon } \\
\text { Mass (g) }\end{array}$} & \multicolumn{2}{|c|}{$\begin{array}{c}\text { Initial Dye } \\
\text { Conc. } C_{o}\left(\mathrm{mg} / \mathrm{dm}^{3}\right)\end{array}$} & \multirow{2}{*}{\multicolumn{2}{|c|}{$\begin{array}{c}\begin{array}{c}\mathrm{AB} 80 \\
k_{f, I}=2.94 \times 10^{-4} \\
\mathrm{~cm} / \mathrm{s}\end{array} \\
D_{s, 1}\left(\mathrm{~cm}^{2} / \mathrm{s}\right)\end{array}$}} & \multirow{2}{*}{$\begin{array}{c}\mathrm{AY} 117 \\
k_{f, 2}=5.04 \times 10^{-4} \\
\mathrm{~cm} / \mathrm{s}\end{array}$} & \multirow{2}{*}{$\begin{array}{c}\text { SSE (AB80+ } \\
\text { AY117) } \\
\left(10^{-6}\right)\end{array}$} \\
\hline & $\mathrm{AB} 80$ & AY117 & & & & \\
\hline 1.7 & 25 & 25 & $8.00 \times 10^{-11}$ & & $6.04 \times 10^{-11}$ & 1.483 \\
\hline 1.7 & 37.5 & 37.5 & $1.20 \times 10^{-10}$ & 콩 & $9.04 \times 10^{-11}$ & 5.110 \\
\hline 1.7 & 50 & 50 & $1.51 \times 10^{-10}$ & $\begin{array}{l}\mathbb{D} \\
\mathbb{0} \\
\mathrm{n}\end{array}$ & $1.18 \times 10^{-10}$ & 5.184 \\
\hline 1.7 & 75 & 75 & $2.15 \times 10^{-10}$ & ๑) & $1.69 \times 10^{-10}$ & 6.394 \\
\hline 1.7 & 100 & 100 & $2.70 \times 10^{-10}$ & & $2.04 \times 10^{-10}$ & 26.712 \\
\hline 0.4 & 50 & 50 & $1.43 \times 10^{-10}$ & $\frac{D}{3}$ & $1.26 \times 10^{-10}$ & 1.612 \\
\hline 0.8 & 50 & 50 & $1.49 \times 10^{-10}$ & 驾 & $1.23 \times 10^{-10}$ & 1.611 \\
\hline 1.2 & 50 & 50 & $1.48 \times 10^{-10}$ & 守 & $1.22 \times 10^{-10}$ & 4.495 \\
\hline 1.7 & 50 & 50 & $1.51 \times 10^{-10}$ & \begin{tabular}{|l|} 
gू \\
3 \\
\end{tabular} & $1.18 \times 10^{-10}$ & 5.184 \\
\hline 2.2 & 50 & 50 & $1.45 \times 10^{-10}$ & & $1.20 \times 10^{-10}$ & 9.986 \\
\hline
\end{tabular}

\section{Batch Kinetic Studies (Results and Discussion)}

- For the AB80+AY117 system, the $D_{s}$ values of $A B 80$ in the single component system are smaller than the $D_{s, 1}$ values of AB80 in the AB80+AY117 binary system while the $D^{--_{s}}$ values of AY117 produce an opposite trend, the $D_{s}$ values of AY 117 in the single component system are greater than the $D_{s, 2}$ values of AY117 in the AB80+AY117 binary system. 


\section{Batch Kinetic Studies (Results and Discussion)}

- A Higashi expression (Higashi and Oishi, 1963) has been used to correlate the relationship between the multicomponent surface diffusivities, $D_{s, 1}$ and $D_{s, 2}$, and the fractional coverage, $\theta_{A}$ and $\theta_{B}$, of the acid dyes in the binary adsorption system. The expressions are shown in equations 18 and 19 and the plots are shown in Figure 6:

where $D_{1}$ and $D_{2}$ are the self-diffusivities for components 1 and 2 in the mixture.

\section{Batch Kinetic Studies (Results and Discussion)}

- It was found that the surface diffusivities are linearly proportion to the fractional coverage expressions and are similar in trend with the findings in the single component HSDM (see Figure 6 and Table 3).

Table 3: Slope and y-Intercept Values of the Correlated Lines in the Plots of the Surface Diffusivity against Fractional Coverage Expression

\begin{tabular}{|c|c|c|c|}
\hline \multirow{2}{*}{ Dye } & System & Slope & y-intercept \\
\hline \multirow{2}{*}{ Acid Blue 80 } & AB80 (single) & $2.646 \times 10^{-9}$ & $2.686 \times 10^{-9}$ \\
\cline { 2 - 4 } & AB80+AY117 & $1.449 \times 10^{-10}$ & $1.524 \times 10^{-10}$ \\
\hline \multirow{2}{*}{ Acid Yellow 117 } & AY117 (single) & $2.702 \times 10^{-9}$ & $2.896 \times 10^{-9}$ \\
\cline { 2 - 4 } & AB80+AY117 & $2.525 \times 10^{-9}$ & $2.566 \times 10^{-9}$ \\
\hline
\end{tabular}


Figure 6: The Plots of Surface Diffusivity against Fractional Coverage

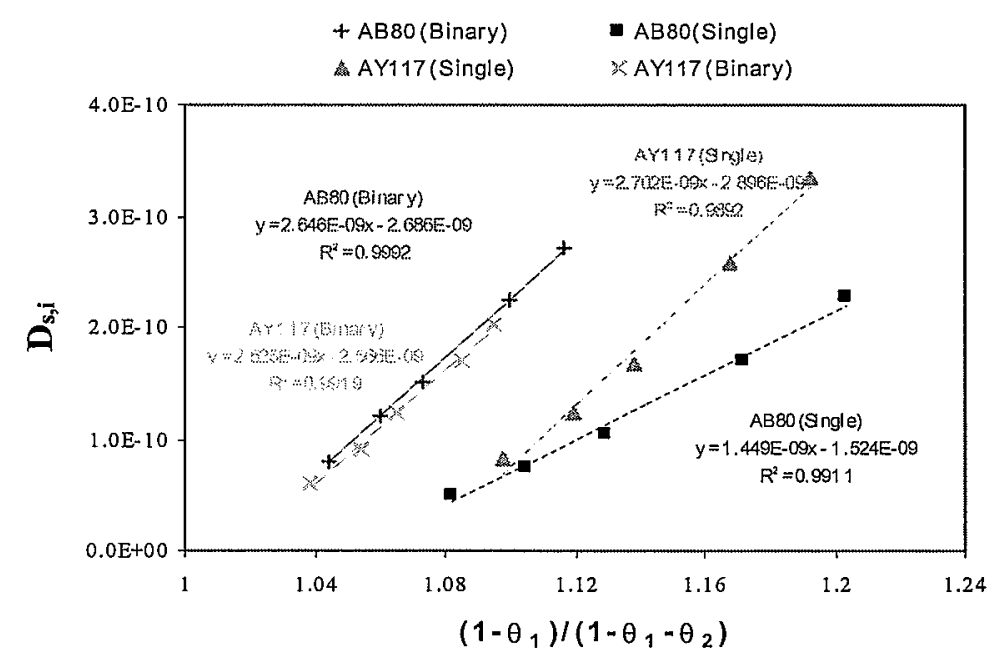

\section{Conclusions}

For the Equilibrium Isotherm Studies:

- The Redlich-Peterson isotherm equation was found to provide the good correlation for the sorption of the two acid dyes, AB80 and AY117, on activated carbon.

For the Batch Kinetic Studies:

- A Multicomponent HSDM was developed by incorporating the HSDM with the IAST model using the Redlich-Peterson isotherm equation.

- The Multicomponent HSDM described the experimental data with a high degree of accuracy for a wide range of carbon mass and initial dye concentration systems.

- The multicomponent surface diffusivities were found to be strongly dependent on the effect of initial dye concentration.

- They are linearly proportion to the fractional coverage expressions and are similar in trend with the findings in the single component HSDM. 


\section{Acknowledgements}

- The authors would like to thank the RGC and DAG (Hong Kong SAR) for providing support of this research programme. 